\title{
Evaluación del Poder Antioxidante de Fracciones de Aceite Esencial Crudo de Schinus molle L. obtenidas por Destilación al Vacío
}

\author{
María S. Guala ${ }^{1}$, Heriberto V. Elder ${ }^{2}$, Gustavo Perez ${ }^{1}$ y Alejandro Chiesa ${ }^{1}$ \\ (1) Universidad Nacional del Litoral, Facultad de Ingeniería Química, Santiago del Estero 2654, \\ Santa Fe-Argentina (e-mail: mguala@fiq.unl.edu.ar) \\ (2) Min. de la Producción-CONICET-UNL, Programa de Plantas Aromáticas y Medicinales, \\ Ruta Provincial 2 №2400, Monte Vera, Santa Fe-Argentina
}

\begin{abstract}
Resumen
Se han evaluado las características antioxidantes del aceite esencial crudo de Schinus molle L. (molle, aguaribay), y se han comparado con las fracciones del mismo aceite obtenidas por destilación al vacío. Las muestras obtenidas se analizaron mediante cromatografía GS/MS, y se les determinó la eficiencia antioxidante utilizando el método DPPH, 2,2 Diphenyl 1 picrylhydrazyl. La fracción más pesada contiene mayor cantidad de Terpinen-4-ol y Germacreno D, compuestos colectores de radicales libres. Por lo tanto, la fracción más pesada es la que tiene mayor poder antirradical.
\end{abstract}

Palabras clave: poder antioxidante, aceite esencial, Schinus molle L., destilación al vacío

\section{Evaluation of the Antioxidant Power of Fractions of Schinus molle L. Essential Oil obtained by Vacuum Distillation}

\begin{abstract}
The antioxidant characteristics of the Schinus molle $L$ (molle, aguaribay) raw essential oil were evaluated and compared with different cuts from the same oil obtained by vacuum distillation. The samples were analysed using GS/MS chromatography and the antioxidant efficiency was determined using the DPPH method, 2.2 Diphenyl 1 picrylhydrazyl. An analysis of the results we may conclude that the fraction is the heaviest it has greater power antiradical. The results show that the heaviest fraction contains higher concentration of Terpinen-4-ol and Germacreno D, compounds that are collectors of free radicals. Therefore, the heaviest fraction has greater antiradical power.
\end{abstract}

Keywords: antioxidant power, essential oil, Schinus molle L., vacuum distillation 


\section{INTRODUCCIÓN}

La oxidación de los productos alimenticios, cosméticos y medicinales, produce un deterioro muy significativo en los mismos, haciendo que pierdan, la mayoría de las veces las propiedades para las cuales fueron elaborados. Las grasas y aceites presentes en estos productos, pueden deteriorarse fácilmente debido a la oxidación, en una serie de reacciones con participación de radicales libres, propagándose y finalmente convirtiéndose en compuestos estables oxigenados, que conllevan a la pérdida de aroma y sabor, junto con otras características indeseables que degradan la calidad del producto (Shahidi, 1997).

Para prevenir tales reacciones se utilizan compuestos con propiedades antioxidantes, en su amplia mayoría sintéticos, como ser Butilhidroxitolueno (BHT), Butilhidroxianisol (BHA), Etilhidroxibenzoato (EHB), TBC, Galato de n-Propilo, por mencionar algunos. Últimamente ha crecido la demanda de "productos de origen natural", libres de aditivos químicos sintéticos, y se ha incrementado el estudio y el uso de sustancias que contengan antioxidantes naturales. Además, está en auge el mercado de complementos dietéticos y nutraceúticos que contengan compuestos capaces de inhibir o inactivar radicales libres, ya que estos compuestos han sido relacionados con enfermedades crónicas y degenerativas, como cáncer, enfermedades neuronales y cardiovasculares (González-Laredo, et al., 2007). Algunos autores han informado sobre las características antioxidante de distintos aceites esenciales crudos (AEC), como ser los de Rosmarinus officinalis L. (Romero) (Martinello y Pramparo, 2005), de Thymus vulgaris L. (Tomillo), de Origanum vulgare L. (Orégano) (Bozin et al., 2006) y Lippia graveolens Kunth (Orégano mexicano) (Martinez-Rocha et al., 2008). Los AEC presentan gran variabilidad en su composición química según la ubicación geográfica de la especie vegetal de la cual se los extrae, ya que inciden las características del clima, y del suelo en su desarrollo.

De todo lo mencionado anteriormente surge la importancia de obtener productos naturales con características antioxidantes a partir de especies vegetales de la región. El AEC que se analiza en el presente trabajo es el de Schinus molle L. (molle, aguaribay), especie vegetal de la familia Anacardiácea, nativa de América del Sur, ampliamente difundida en la zona del Litoral Argentino.

La mayoría de las publicaciones recientes se focalizan en el estudio de las características antioxidantes de los aceites esenciales crudos, sin embargo, en la literatura no hay información respecto de la conveniencia o no de fraccionar los mismos. Por lo que el objetivo de este trabajo es comparar las características antioxidantes del aceite esencial crudo y de los distintos cortes obtenidos por destilación al vacío, a fin de determinar si algún grupo de compuestos presenta mayores características antioxidantes que otro.

\section{MATERIALES Y MÉTODOS}

Se detalla a continuación los materiales y la metodología utilizada para la obtención del AEC y sus fracciones, la técnica analítica utilizada para evaluar la capacidad antioxidante de las distintas muestras, y el análisis estadístico realizado a los valores experimentales.

\section{Materia Prima}

El material utilizado para la extracción del AEC se cosechó, durante el mes de noviembre de 2006, de plantas de Schinus molle L., cultivados en el Centro Operativo Experimental "Angel Gallardo (Centro perteneciente al Ministerio de la Producción de la Provincia de Santa Fe, Argentina). La materia prima se preparó a partir de frutos maduros molidos, hojas y tallos pequeños con un diámetro no mayor a 0,4 - 0,6 cm. El aceite esencial se obtuvo por hidrodestilación mediante un equipo Clevenger.

Los distintos cortes se obtuvieron por destilación al vacío, utilizando un equipo de escala laboratorio constituido de un balón, refrigerante, y sistema colector de las distintas fracciones. El sistema se conectó a una bomba de vacío y se operó a una presión de $33 \mathrm{mmHg}$, con reflujo. Las fracciones que se obtuvieron y los rangos de temperaturas se muestran en la Tabla 1. 
Tabla 1: Rangos de temperatura a los cuales se obtuvieron los distintos cortes

\begin{tabular}{lc}
\hline Cortes & Rangos de temperaturas \\
\hline Corte 1 & $76^{\circ} \mathrm{C} \leq \mathrm{T}<78^{\circ} \mathrm{C}$ \\
Corte 2 & $78^{\circ} \mathrm{C} \leq \mathrm{T}<81^{\circ} \mathrm{C}$ \\
Corte 3 & $81^{\circ} \mathrm{C} \leq \mathrm{T}<89^{\circ} \mathrm{C}$ \\
Corte 4 & $89^{\circ} \mathrm{C} \leq \mathrm{T}<106^{\circ} \mathrm{C}$ \\
Residuo & $106^{\circ} \mathrm{C}>\mathrm{T}$ \\
\hline
\end{tabular}

\section{Determinación analítica}

La capacidad antioxidante de las distintas muestras se evaluó como la capacidad de atrapar radicales utilizando la técnica del radical libre 2,2-Diphenyl-1-picrylhydrazyl (DPPH), siguiendo la metodología propuesta por Brand-Williams et al. (1995), con las modificaciones de Sánchez-Moreno et. al. (1997). Se eligió esta técnica por su simplicidad, y precisión (Shahidi, 1997), y además porque el objetivo es comparar las distintas muestras, provenientes de un mismo aceite y no obtener valores absolutos.

Se usó metanol grado analítico Anedra y 2,2-Diphenyl-1 -picrylhydrazyl (DPPH) Sigma - Aldrich. El espectrofotómetro utilizado es marca Jenway, modelo 6305, UV - visible, con un rango de longitudes de onda de 198 - $1000 \mathrm{~nm}$, una resolución de $1 \mathrm{~nm}$ y una exactitud de \pm 2 .

La capacidad antioxidante fue expresada como valores de "Eficiencia Antioxidante" (EA) definida como:

$E A=1 /\left(E_{50} \cdot \mathrm{T}_{\mathrm{EC} 50}\right)$

Siendo $\mathrm{EC}_{50}$ : la cantidad de AEC necesaria para disminuir la concentración de DPPH inicial al 50\% y $\mathrm{T}_{\mathrm{EC} 50}$ : el tiempo necesario para alcanzar el estado estacionario correspondiente al $\mathrm{EC}_{50}$.

Se prepararon muestras con distintas concentraciones del material a analizar, se tomaron $0,2 \mathrm{ml}$ de las mismas, se les adicionó 7,8 $\mathrm{ml}$ de una solución de DPPH en metanol $(0,05 \mathrm{~g} / \mathrm{l})$, y se realizó un seguimiento de la variación de la absorbancia en función del tiempo utilizando un espectrofotómetro. A partir de estas evoluciones se graficaron los valores de DPPH y del tiempo, a estado estacionario, en función de los gramos de antioxidante por gramo de DPPH, para obtener el $E_{50}$ y el $T_{E C 50}$, y la EA. Previamente, se debió calibrar el equipo, para lo cual se construyó una curva de absorbancia en función de concentración de DPPH. La técnica fue validada midiendo la EA de la quercitina y comparándolo con valores publicados en la literatura.

Las muestras se analizaron por GC/MS empleando equipo GC Hewlett Packard 6890 con un detector MS HP 5972. La identificación de los componentes se realizó a partir de sus espectros de masas, los índices de retención y los índices de Kovats, comparando con datos de la literatura (Adams, 1995), con compuestos testigos o con datos propios. Se utilizó una columna capilar de sílice fundido, con un largo $60 \mathrm{~m}$ y un diámetro interno de $0,25 \mathrm{~mm}$; la fase estacionaria de $5 \%$ fenil-95\% metil silicona, con un espesor de $0,25 \mu \mathrm{m}$; la temperatura en el horno de $90{ }^{\circ} \mathrm{C}$, luego a $3{ }^{\circ} \mathrm{C} / \mathrm{min}$ hasta $225^{\circ} \mathrm{C}$; la temperatura en el inyector de $255^{\circ} \mathrm{C}$; la temperatura del detector $275^{\circ} \mathrm{C}$; el detector FID; el gas de arrastre helio.

\section{Análisis estadístico}

Los valores experimentales se informan como valores medios \pm la Desviación Standard (DS) de tres muestras. Las curvas fueron correlacionadas con modelos exponenciales dando coeficientes de correlación $p>0.99$.

\section{RESULTADOS Y DISCUSIÓN}

A titulo ilustrativo se muestran los gráficos realizados para determinar la EA, solamente para el aceite esencial crudo, se procedió de la misma para las otras muestras. En la figura 1 se muestra la 
evolución del porcentaje de DPPH remanente (\%DPPHr), definido como el DPPH remanente sobre el DPPH inicial por ciento, en función del tiempo para muestras de distinta concentración de aceite esencial crudo. La figura 2 contiene el \%DPPHr, a estado estacionario, en función de los gramos de antioxidante por gramo de DPPH. A partir de esta gráfica se obtiene el valor de EC $_{50}$. La figura 3 muestra la variación del tiempo necesario para alcanzar el estado estacionario en función de la concentración de AEC. A partir de esta gráfica se obtiene el $T_{E C 50}$. Los valores obtenidos para el AEC y los cortes se consignan en la Tabla 2. Los valores para los cortes 1 y 2 no se determinaron debido a que la evolución era sumamente lenta y no se llegó a los valores a estado estacionario.

Los componentes de las distintas muestras, obtenidos por cromatografía, se muestran en la tabla 3.

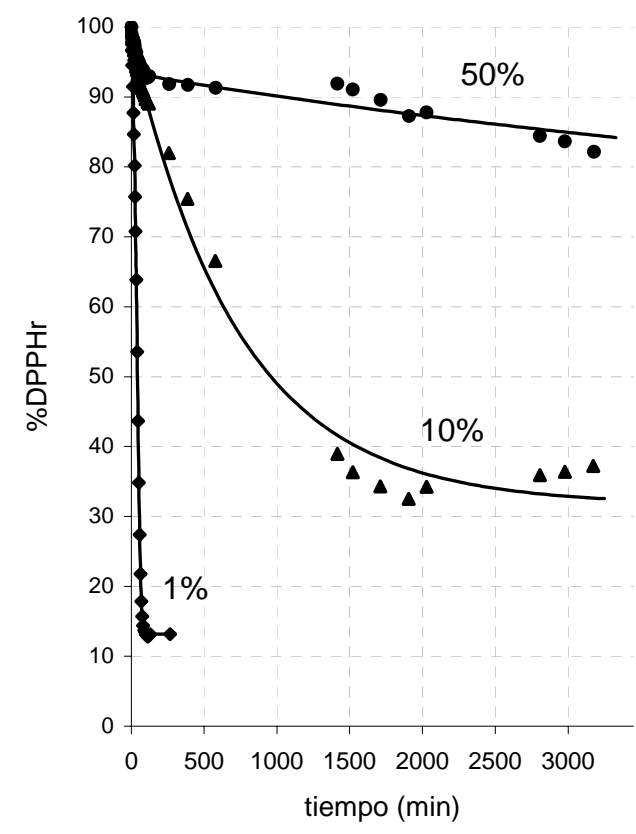

Fig. 1: Evolución del \%DPPHr para el AEC

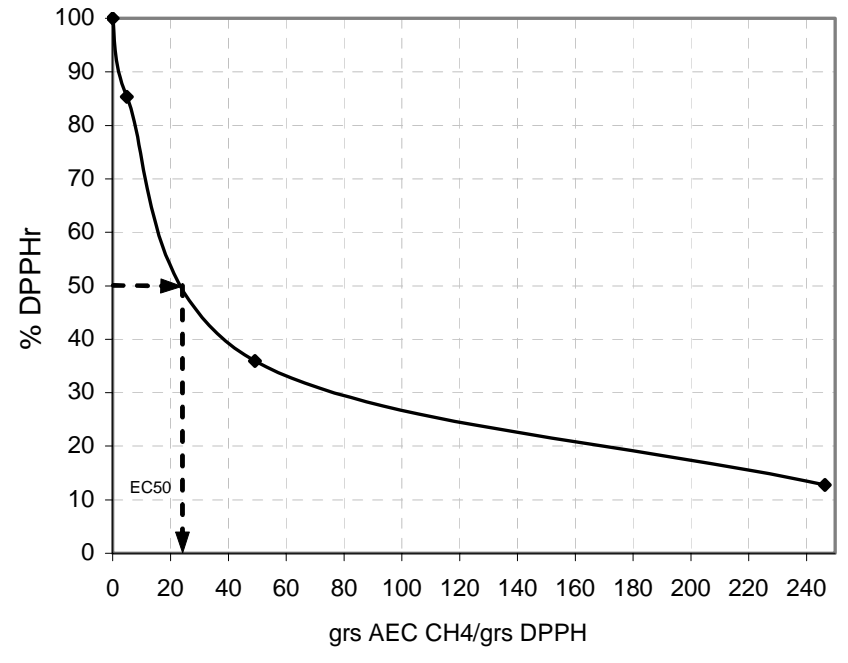

Fig. 2: \%DPPHr vs concentración de antioxidante

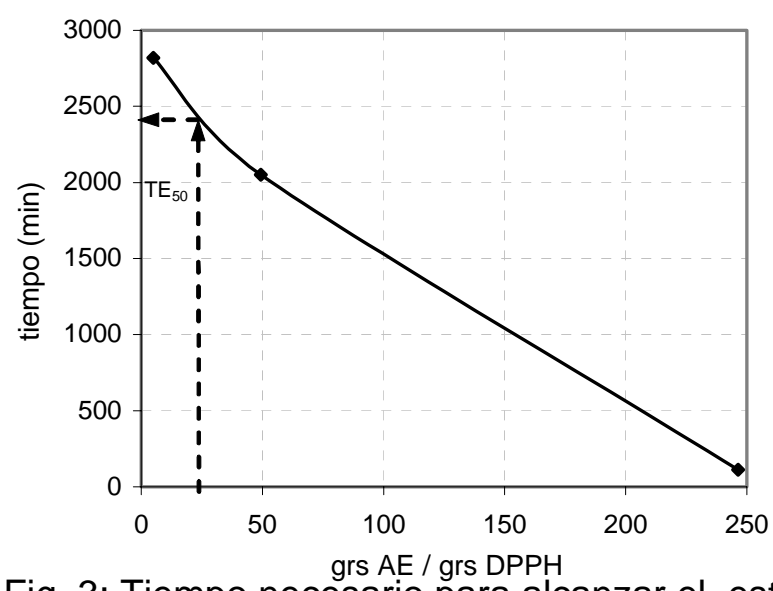

Fig. 3: Tiempo necesario para alcanzar el estado estacionario vs la concentración de AEC

La eficiencia antioxidante (EA) es mayor cuando menor es la cantidad de antioxidante que se necesite (menor $\mathrm{EC}_{50}$ ), y cuando la reacción alcance antes el estado estacionario (menor $\mathrm{T}_{\mathrm{EC} 50}$ ). $\mathrm{Si}$ bien, los valores de los cortes 1 y 2 no se muestran, de las evoluciones se pudo concluir que tendrían valores de EA mayores que el corte 3. Por lo tanto de los valores mostrados en la Tabla 2, y con la aclaración anterior, se puede ver que el residuo es el que mayor EA posee. 
Tabla 2: Valores de $\mathrm{EC}_{50}, \mathrm{TEC}_{50}$ y EA para los distintos materiales estudiados

\begin{tabular}{lccc}
\hline Material & $\mathrm{EC}_{50}$ (gr. Material/gr. DPPH) & $\mathrm{TE}_{50}(\mathrm{~min})$ & $\mathrm{EA}$ \\
\hline $\mathrm{AEC}$ & $29,13 \pm 0,45$ & $2300 \pm 22$ & $1.499 \mathrm{E}-5$ \\
Corte 3 & $37,93 \pm 0,25$ & $3700 \pm 10$ & $0,711 \mathrm{E}-8$ \\
Corte 4 & $11,30 \pm 0.51$ & $7200 \pm 33$ & $1.260 \mathrm{E}-8$ \\
Residuo & $14,16 \pm 0.45$ & $1150 \pm 18$ & $6.211 \mathrm{E}-8$ \\
\hline
\end{tabular}

Tabla 3: Principales compuestos del AEC y de los distintos cortes

\begin{tabular}{lcccccc}
\hline Componentes & AEC & $\begin{array}{c}\text { Corte } \\
\mathbf{1}\end{array}$ & $\begin{array}{c}\text { Corte } \\
\mathbf{2}\end{array}$ & $\begin{array}{c}\text { Corte } \\
\mathbf{3}\end{array}$ & $\begin{array}{c}\text { Corte } \\
\mathbf{4}\end{array}$ & $\begin{array}{c}\text { Residuo } \\
\text { Balón }\end{array}$ \\
\hline tuyeno & & & & & & \\
alfa-pineno & 1,0 & 2,1 & 1,4 & 0,7 & 0,2 & - \\
canfeno & 0,3 & 0,4 & 0,3 & 0,3 & & - \\
beta-pineno & 12,7 & 16,7 & 16,1 & 16,5 & 15,4 & 2,1 \\
sabineno & 45,0 & 52,4 & 54,0 & 60,0 & 52,4 & 7,3 \\
mirceno & 2,4 & 1,8 & 1,6 & 3,0 & 4,5 & 1,1 \\
alfa terpineno & 1,6 & 0,9 & 1,1 & 0,2 & 3,1 & 1,7 \\
limoneno & 3,8 & 1,8 & 2,8 & 3,9 & 7,5 & 5,8 \\
beta felandreno & 1,3 & 0,6 & 0,8 & 1,0 & 2,5 & 1,8 \\
gamma & 2,9 & 0,7 & 1,2 & 0,5 & 4,4 & 7,0 \\
terpineno & & & & & & \\
p-cimeno & 1,0 & 0,5 & 1,0 & 2,9 & 2,0 & 1,9 \\
terpinoleno & 0,6 & 0,0 & 0,2 & 0,0 & 0,7 & 1,9 \\
Terpinen-4-ol & 4,4 & 0,3 & 0,3 & 0,4 & 0,9 & 19,5 \\
beta-cariofileno & 1,2 & - & - & - & - & 5,4 \\
alfa humuleno & 0,2 & - & - & - & - & 0,7 \\
alfa terpineol & 0,1 & - & - & - & - & 0,6 \\
germacreno D & 1,0 & - & - & - & - & 4,1 \\
tau cadinol & - & - & - & - & - & 7,8 \\
TOTAL & 91,2 & 100,0 & 96,6 & 98,2 & 96,8 & 68,7 \\
\hline
\end{tabular}

A partir del análisis de los componentes del AEC y de sus fracciones se observa que la fracción más pesada contiene mayores cantidades de Terpin-4-ol, y de Germacreno D. El Terpinen-4-ol, se ha informado en la literatura como una sustancia antioxidante (Suhaj, 2004; Hyun-Jin et al. 2004) y también el Germacreno D (Juteau et. al., 2002; Souza et al., 2007).

\section{CONCLUSIONES}

Los resultados obtenidos arrojaron que el fraccionamiento del AEC posibilitó obtener un corte con mejores características antioxidantes que el AEC, esto no siempre es así ya que a veces existe sinergia entre los compuestos que hacen que al separarlos se empeoren las características que se quieren potenciar. Que la fracción más pesada es la que tiene mayor poder antirradical, y que puede deberse a que contiene mayor cantidad de Terpinen-4-ol, que es un alcohol no saturado con un doble enlace que lo califica como un potencial secuestrante de radícales libres y de Germacreno D que es altamente reactivo y capturador de radicales libres.

Por lo tanto, puede concluirse que el fraccionamiento de los aceite esenciales crudo puede ser un procedimiento interesante para potenciar las características antioxidantes de los mismos, permitiendo, además, un mejor conocimiento de cuales compuestos contribuyen a mejorar la capacidad de atrapar radicales. 


\section{AGRADECIMIENTOS}

A la Universidad Nacional del Litoral por el apoyo financiero a través de la programación CAl+D. Programa CYTED (Proyecto: Promoción del Desarrollo de Agroindustrias no Tradicionales Dedicadas a Recursos Vegetales Iberoamericanos) (306RT0278).

\section{REFERENCIAS}

Adams, R.; Identification of Essential Oil Components by Gas Chromatography / Mass Spectroscopy, $2^{\text {da }}$ ed., Allured Publishing Corporation, Illinois, USA (1995).

Brand-Williams W., M. Cuvelier y C. Berset; Use of a Free Radical Method to Evaluate Antioxidant Activity, Lebensm. Wiss. U. Technol.: 28, 25-30 (1997).

Bozin B., N. Mímica-Durkin, N. Simin y G. Anacrov; Characterization of Volatile Composition of Essential Oils of Some Lamiaceae Spices and Antimicrobial and Antioxidant Activities of the Entire Oils, J. Agric. Food Chem.: 54, 1822-1828 (2006).

González-Laredo R. y otros cinco autores; Evaluación del efecto antioxidante y quimioprotector de extractos fenólicos de semillas de manzana, Grasas y Aceites: 58 (1), 5-9, (2007).

Hyun-in K., W. Feng, W. Changqing y W. Xi; Evaluation of antioxidant activity of Australian Tea Tree (Melaleuca alternifolia), oils and its components, J.Agric. Food Chem.: 52 (10), 2849-2854 (2004).

Juteau, F. y otros cuatro autores; Antibacterial and antioxidant activities of Artemisia annua essential oils, Fitoterapia: 73 (6), 532-535 (2002).

Martinello M. A. y M. Pramparo; Poder antioxidante de extractos de romero concentrados por destilación molecular, Información Tecnológica: 16 (5), 17-20 (2005).

Martínez-Rocha A. y otros cuatro autores; Antioxidant and Antimutagenic Activities of Mexican Oregano (Lippia graveolens Kunth); Plant Foods Hum. Nutr.: 63, 1-5 (2008).

Sánchez-Moreno C., J. Larrauri y F. Saura-Calixto; A procedure to measure the antiradical efficiency of polyphenols, J. Sci. Food Agric.: 67, 415-420 (1997).

Shahidi, F.; Natural antioxidants: chemistry, health effects and applications. AOCS Press. Champaign, Illinois, EE.UU (1997).

Souza, T.J., M. Apel, S. Bordignon y N. Matzenbacher; Chemical composition and antioxidant activity of the volatile oil from Eupatorium polystachyum DC, Revista Brasileira de Farmacognosia: 17(3) (2007)

Suhaj M.; Spice antioxidants isolation and their antiradical activity: a review. Food Research Institute, Bratislava, Eslovaquia (2004). 